\title{
On the Application of Psychological Training in Athletic Training
}

\author{
$\mathrm{Bin} \mathrm{Xu}$ \\ College of Foreign Languages, Northeast Dianli University, Jilin, 132012, China
}

\begin{abstract}
Humans have senior intelligence which general animals do not have it is necessary to use human intelligence furthest, with a higher standard to consider our sports training. The article research and discuss the application of the psychological suggestion training in sports training in psychology.The results show that introducing psychology to sport training can not only improve the training effect, and can promote the sports training to a higher level. Of course, psychological suggestion is supplementary mean in sports training. Only linking training of psychological suggestion and the actual training closely together, can obtain good results.
\end{abstract} training

Keywords-psychological suggestion training, sports

\section{PSYCHOLOGICAL SUGGESTION TRAINING PSYCHOLOGY TRAINING}

Psychological suggestion refers to the reflection of image of the human sense of the past things, which is the basis of sensation and perception, is the trace of the brain during the perception process. So the object that psychology suggests reflected is not through the current directly organ, but is senses of the trace in the brain in the past .Psychology training is depicted own sports in the brain, using the means of seeking memory, recognize the actual sports process of muscle contraction again, so that to achieve it have the same effect as the actual sports muscle contraction.

According to the theory of sports memory, there are two big systems to control our reflection memory: one is through sports, the body of human's sensory memory; The second one is using the form of memory. The two big system are closely linked to a series of memory of human about the language, so they are called the series of "language" memory. human sensory memory, is describing the sports things have experienced, so that human body's nerve endings changed, and many research results have proved that.

For example using the bio-electric phenomenon, Japanese Sport Psychologist, zhuwugong hong regarded the EMG as his indexes, use jacobson to show the man's situation of psychological suggestion according to his own sport experience. He let the man lay supine and be relaxed, when he describe a special ports, the man's muscle contraction would change, the EMG would show the change, and then compare this change to the actual situation, the result show that the man's muscle contraction changed strongly when described a special sport, even more stronger than the actual sports.
Another Japanese sports psychologist Fujita EEG, takes electroencephalography, eye movement, movement of the limbs electrocardiograph, pulse breathing as his indicators, such as further by comprehensive methods, gymnastics, swimming, skiing and other athletes as test object, let them be engaged in the most good at sports psychological hint, and then record them. Results also showed the same as the actual movement of regular reflect the test pattern, especially appeared on the alpha blockade like now, on the pulse and breathing test pattern also appeared obvious with the different reflection of relaxation. They provide tables as the reliability of the training effectiveness in learning.

\section{THE VALUE OF THE APPLICATION OF PSYCHOLOGY TRAINING}

We human beings have high level of intelligence that generally animals do not have. To maximize the use of human intelligence, with a higher standard to consider our sports is so necessary. Applied psychology suggests that training be mostly used to control or to correct a technology or movement skills, it didn't realize that implement to coincide with the actual movement of mental training. In fact, if we can combine mental training with the actual close touch in sports training, interaction, its effect is better than just take actual practice with good effect. A simple example, before the speech in the official, if in advance can carefully prepared to speak and repeated drills, formal speech, its effect and rehearse beforehand speech effect is much better. The reason is very simple, the former has had on the basis of sensation and perception about things. So, on the basis of the actual movement training, closely combined with the mental training, will no doubt greatly improve the training effect.

\section{THE APPLICATION OF PSYCHOLOGICAL TRAINING IN} ATHLETIC TRAINING

During application of psychological suggestion in training, a very important point is how imagery depicted in one's mind. Usually, movement psychological hinted of depicting General can be divided into two categories: one is in the process of master or correct all kinds of sports skills, it will be a technical action according to the actual correct action for paint in your mind, to achieve the degree that control freely, thus improve the technical level; Another kind is in the whole training (or race), to eliminate tension and uneasiness in movement, enhanced ability to adapt and in training or competition can give full play to be adopted by the technical level of a psychological training methods. In terms of the first 
category, athletes in sports practice has been more or less contact, and we will focus on the second category to describe. In the process of portraying second category we must put the actual scene to depict the training or competition, in your mind by preparing activities start to the end of the training group (or ratio), repeatedly rehearsed in your mind. Such as: about sprint training in psychology to allude, from start to cross the finish line; Throwing image training from entering throwing area to missiles from the hands of the whole process of repeated drills. At the same time in the psychological portray must also do our best to describe in detail the body (or race) in the actual training posture, strength and speed, etc. Through such repeated mental training, even to the actual training or competition, not nervous, and can be composed freely play their proper level.

There are some kinds specific application of psychological insinuation training, as follows:

Before the training

We should first clear the content and purpose of practical training. And then carry out in accordance with the order of practical training content representation. Psychological depiction should not only use visual sense to appearance posture, conducted by themselves according to the time order like a bed of the drills in the brain, but also use the rhythm of the kin aesthetic, will complete the action, the degree of muscle force and so on. We should completely according to the actual action to practice of requirements, but also the emergency in position should be taken into account, such as: all kinds of noise, his, such as changes in the weather. Only in this way can the training of psychological suggestion be closely connected to the actual training, adapt to each kind of situation on the position of change, in order to gain better training results.

In the training

Before preparing sports activities, we should first to mercerizing the content and the purpose of practice in advance in your mind, then to start to prepare it. Activities can also be more ready to mental training (the content of the image can choose according to particular case ), which is not only beneficial to the right move for consolidate and improve, but also can arouse the enthusiasm for the athletes' training. When into the main content of the training, according to the order, do a psychological description full into action again after practice. If it is a single technology as an exercise, can exercise a rehearsal time, interaction into the line. Practice should pay special attention to find the actual practice and psychological differences, find problems and solve in time. If it cannot be solved immediately, can find out the reason, first for training, after the analysis.

After the training

After the end of training the human body have become a bit quiet, we should settle down the content of the practice in the brain comprehensive on the same day, focusing on the actual practice with psychological describe error on the content of the careful analysis, whether it is a technical action problem or a mental picture out of my practical ability, can also be widely listen to the views of his teammates and coaches, conditional word can also use video, put himself in the actual practice in the case of a psychological describe content, providing the basis for the next session. Of course due to the nature of the various sports and there are some differences, and have different mental training in the practical application, these must according to the actual situation of each person to make adept flexibility.

\section{CONCLUSIONS}

In order to reach a higher level, athletes in sports training at the same time, should do correct mental training. This is significant to promote the level of training. Moreover in the process of mental training, we must concentrate on the images of psychological suggestion, it can be received in training or competition thought highly and have the good effects. Mental training, of course, is an auxiliary means, it is only for the improvement of technology has a positive role, therefore, the mental training practice with the actual movement must be closely linked, and insist on not slack, then we can get the result that beyond our expecting.

\section{CORRESPONDING AUTHOR}

Bin $\mathrm{Xu}$. College of Foreign Languages, Northeast Dian li University, Jilin, 132012, China. Email:34063479@qq.com.TEL:15843294007

\section{REFERENCES}

[1] Qin Zhihui: "to explore the" problems of endurance training in football players, "Journal of Beijing Sport University" 2002.25 (1):136-138.

[2] Geng Jianhua: "analysis and training countermeasures" structure of physical training at the present stage of football players in China, "Journal of Wuhan Sports Institute, 2004.38 (5): 111-113.

[3] Chen Ming: "physical training and eliminate fatigue" football "," Journal of Beijing Sport University, 2006.29 (2): 206-208.

[4] Liu Fuli, Zhou Yi: "analysis" means occupation football team trainingsystem and its components, "Journal of Guangzhou Sports University,2002.22 (1): 68-71. 